\title{
Budesonide/Formoterol Fumarate Dihydrate Inhalation Aerosol
}

National Cancer Institute

\section{Source}

National Cancer Institute. Budesonide/Formoterol Fumarate Dihydrate Inhalation

Aerosol. NCI Thesaurus. Code C71534.

An inhalation aerosol formulation containing budesonide and the fumarate dihydrate salt of formoterol with anti-inflammatory and bronchial smooth muscle-relaxing activities. The synthetic corticosteroid steroid budesonide binds to intracellular glucocorticosteroid receptors (GRs), exhibiting inhibitory activities against multiple cell types and mediators associated with allergic inflammation. The long-acting beta-adrenergic receptor agonist formoterol selectively binds to beta-2 adrenergic receptors in bronchial smooth muscle, activating intracellular adenyl cyclase, an enzyme that catalyzes the conversion of adenosine triphosphate (ATP) to cyclic-3',5'-adenosine monophosphate (cAMP); increased intracellular CAMP result in the relaxation of bronchial smooth muscle and inhibition of the cellular release of mediators of immediate hypersensitivity, especially from mast cells. 